\title{
FINITELY PRESENTED LATTICES: CANONICAL FORMS AND THE COVERING RELATION
}

\author{
RALPH FREESE
}

\begin{abstract}
A canonical form for elements of a lattice freely generated by a partial lattice is given. This form agrees with Whitman's canonical form for free lattices when the partial lattice is an antichain. The connection between this canonical form and the arithmetic of the lattice is given. For example, it is shown that every element of a finitely presented lattice has only finitely many minimal join representations and that every join representation can be refined to one of these. An algorithm is given which decides if a given element of a finitely presented lattice has a cover and finds them if it does. An example is given of a nontrivial, finitely presented lattice with no cover at all.
\end{abstract}

This paper studies finitely presented lattices. We introduce a canonical form for the elements of such a lattice which agrees with Whitman's canonical form for free lattices in the case the finitely presented lattice is free. This canonical form, like Whitman's, has several nice theoretical properties. In addition it allows one to efficiently calculate in such a lattice. We have programs, written in both CoMmon LisP and MULisP, for dealing with finitely presented lattices. We also investigate the covering relation in finitely presented lattices. We show that there is an effective proceedure for determining if an element of such a lattice has any lower covers and for finding them if there are any.

Alan Day [2] has shown that every finitely generated free lattice is weakly atomic, i.e., every interval contains a covering. It was conceivable that such a theorem could extend to all finitely presented lattices. We show that this is not the case. In fact we show that there is a (nontrivial) finitely presented lattice without any coverings.

There is a close connection between finitely presented lattices and lattices freely generated by a finite partial lattice (see below). Because of this, most of our theorems will be phrased in terms of $F L(P)$, the free lattice generated by the partial lattice $\mathbf{P}$.

Received by the editors July 14,1988 .

1980 Mathematics Subject Classification (1985 Revision). Primary 06B25, 06B05. lattice.

Key words and phrases. Finitely presented lattice, covering relation, canonical form, partial

This research was partially supported by NSF grant no. DMS-8521710. 


\section{Preliminaries}

For lattice theory we use the notation of Crawley and Dilworth [1] and for algebra McKenzie, McNulty, and Taylor [10]. We now review certain notions particularly important for this paper. Let $\mathbf{L}$ be a lattice and let $a, b \in L$. We say that $a$ is covered by $b$ and that $b$ covers $a$ if $a<b$ and there is no $c$ with $a<c<b$. We use the notation $a \prec b$ and $b \succ a$. If $b \leq a$ we let $a / b=\{x \in L: b \leq x \leq a\}$ denote the quotient or interval between $b$ and $a$. A lattice is weakly atomic if every nontrivial interval contains a two-element interval.

For any lattice $\mathbf{L}$ and $x, y, z \in L$,

$$
x \vee(y \vee z)=(x \vee y) \vee z .
$$

For efficiency it is best to use the expression $x \vee y \vee z$ to represent this term. Because of this we define a lattice term over the variables $X$, and the rank of such a term, in a slightly unusual manner. Each $x \in X$ is a term of rank 1 . If $t_{1}, \ldots, t_{n}$ are lattice terms with ranks $r_{1}, \ldots, r_{n}$ then $\left(t_{1} \vee \cdots \vee t_{n}\right)$ and $\left(t_{1} \wedge \cdots \wedge t_{n}\right)$ are terms of rank $1+r_{1}+\cdots+r_{n}$. When we write a term we usually omit the outermost parentheses. Notice that both the terms displayed above have rank 5, but $x \vee y \vee z$ has rank 4. Thus our ranking scheme gives preference to the term $x \vee y \vee z$ over either of the terms displayed above. Analogous to this, we sometimes view a lattice $L$, with a least and greatest element, as a set with two functions $\bigvee$ and $\Lambda$ mapping the finite subsets of $L$ into $L$, rather than an algebra with two binary operations. The set of subterms of a term $t$ is defined in the usual way: if $t$ is a variable then $t$ is the only subterm of $t$. Recursively, if $t=t_{1} \vee \cdots \vee t_{n}$ or $t_{1} \wedge \cdots \wedge t_{n}$ then the subterms of $t$ consist of $t$ together with the subterms of $t_{1}, \ldots, t_{n}$.

A lattice presentation is a pair $\langle X, R\rangle$ where $X$ is a set and $R$ is a set of relations in the variables $X$. A relation is an equation $s\left(x_{1}, \ldots, x_{n}\right) \approx$ $t\left(x_{1}, \ldots, x_{n}\right)$ where $s$ and $t$ are lattice terms over $X$. The lattice presented by $\langle X, R\rangle$ is a lattice $\mathbf{F}$ together with a map $f: X \rightarrow \mathbf{F}$ such that

$$
s\left(f\left(x_{1}\right), \ldots, f\left(x_{n}\right)\right)=t\left(f\left(x_{1}\right), \ldots, f\left(x_{n}\right)\right)
$$

for all $s \approx t$ in $R$ and if $g: X \rightarrow \mathbf{L}$ is a function such that

$$
s\left(g\left(x_{1}\right), \ldots, g\left(x_{n}\right)\right)=t\left(g\left(x_{1}\right), \ldots, g\left(x_{n}\right)\right)
$$

holds in a lattice $\mathbf{L}$ for each relation $s \approx t$ in $R$ then there is a unique homomorphism $h$ from $\mathbf{F}$ to $\mathbf{L}$ such that $h(f(x))=g(x)$. A finite presentation is one in which both $X$ and $R$ are finite. A finitely presented lattice is a lattice presented by a finite presentation.

A partial lattice $\mathbf{P}$ is a partially ordered set $\langle P, \leq\rangle$ together with two partial functions $\bigvee$ and $\wedge$ from the set of subsets of $P$ into $P$ such that if $p=\bigvee S$ then $p$ is the least upper bound of $S$ in $\langle P, \leq\rangle$, and the dual condition holds. $\mathbf{F L}(\mathbf{P})$ denotes the lattice freely generated by $\mathbf{P}$. More formally, $F \mathbf{L}(\mathbf{P})$ is defined by the property that it is generated by $P$, the joins and meets defined 
in $\mathbf{P}$ agree with those of $\mathbf{F L}(\mathbf{P})$, and any map $f$ from $P$ to a lattice $\mathbf{L}$, which preserves the order of $\mathbf{P}$ and the joins and meets defined in $\mathbf{P}$, can be extended to a lattice homomorphism of $\mathbf{F L}(\mathbf{P})$ to $\mathbf{L}$.

Most of the time we will be working with a fixed partial lattice $\mathbf{P}$. If $s$ and $t$ are terms in $P$ we define $s \leq t$ to mean that the interpretation of $s$ in $F L(P)$ is less than or equal to that of $t . s \approx t$.neans $s \leq t$ and $t \leq s$.

As we mentioned in the introduction, there is a close connection between finitely presented lattices and lattices freely generated by finite partial lattices. Of course if $P$ is finite then $F L(P)$ can be thought of as a finitely presented lattice. Indeed, if $P$ is finite we can associate a presentation on the set of variables $P$ such that if $x \leq y$ in $\mathbf{P}$ then we have the relation $x \vee y=y$ and if $x=\vee X$ in $\mathbf{P}$ then we have the relation $x=x_{1} \vee \cdots \vee x_{n}$, where $X=\left\{x_{1}, \ldots, x_{n}\right\}$, and similar relations for each meet in $\mathbf{P}$.

Conversely, given a finite presentation $\langle X, R\rangle$, let $\mathbf{F}$ be the corresponding finitely presented lattice. Let $P^{\prime}$ be the union of $X$ and the set of all the subterms of the terms occurring in $R$ and let $P$ be the equivalence classes of $P^{\prime}$ under the equivalence relation which identifies terms which are equal in $\mathbf{F}$. We define $\mathbf{P}$ to be the partial lattice on $P$ whose order is inherited from $\mathbf{F}$ and such that $p=p_{1} \vee \cdots \vee p_{n}$ whenever $t=t_{1} \vee \cdots \vee t_{n}$ is in $R$ or is one of the subterms defining $P^{\prime}$ and $p$ is the equivalence class of $t$ and $p_{i}$ is the class of $t_{i}, i=1, \ldots, n$. Of course the meets in $\mathbf{P}$ are defined by duality. The map which sends each $x \in X$ to its equivalence class extends to an isomorphism of F onto $\mathbf{F L}(\mathbf{P})$.

Since the word problem for finitely presented lattices is solvable (see below), the above remarks show that there is a natural (and effective) way to associate with each finite presentation, a partial lattice and vice versa, so that the corresponding lattices are isomorphic. In this paper we will actually study $\mathbf{F L}(\mathbf{P})$ where $\mathbf{P}$ is a finite partial lattice.

\section{DEAN'S SOLUTION TO THE WORD PROBLEM}

P. Whitman [12] gave a recursive procedure for deciding if two terms represent the same element in the free lattice, thus solving the word problem for free lattices. The word problem for finitely presented lattices was shown to be uniformly solvable by J. C. C. McKinsey [11]. Evans [4] also showed the word problem was uniformly solvable as a special case of a more general theorem. Freese and Nation showed that the generalized word problem, and certain other decision problems for lattices, are solvable, [6]. Whitman's solution has a close connection with the arithmetic of the free lattices and in fact most results about free lattices are derived from his solution. R. A. Dean [3] gave a solution to the word problem for finitely presented lattices which is as close as possible to Whitman's solution. In this section we review Dean's solution and some of its consequences.

Dean's (and Whitman's) algorithm tests if $s \leq t$. As we mentioned above, $s \approx t$ if and only if $s \leq t$ and $t \leq s$, so Dean's algorithm can be used to test 
$s \approx t$. Both Dean's and Whitman's algorithm reduce the problem of deciding if $s \leq t$ to a Boolean combination of similar problems involving terms of lower ranks. Dean's algorithm is presented in the next theorem. Recall that for lattice terms $s$ and $t$ with variables from $P, s \leq t$ means that $s^{\mathbf{F L}(\mathbf{P})} \leq t^{\mathbf{F L}(\mathbf{P})}$. By an ideal of a partial lattice $\mathbf{P}$ we mean a subset $I$ such that if $y \leq x \in I$ then $y \in I$ and $I$ is closed under joins of elements of $I$ when they are defined. Finding the ideal generated by a subset $Y$ of a partial lattice is more difficult than with a lattice. One must close $Y$ under existing joins, then add all elements below these, and then repeat this process until nothing new is obtained. Since $P$ is finite, this is effective. For $w \in \mathbf{F L}(\mathbf{P})$, we define $\operatorname{Id}_{\mathbf{P}}(w)=\{x \in P: x \leq$ $w\}$. If $t$ is a term, $\operatorname{Id}_{\mathbf{P}}(t)=\operatorname{Id}_{\mathbf{P}}(w)$, where $w$ is the interpretation of $t$ in $\mathbf{F L}(\mathbf{P})$. If $S \subseteq \mathrm{FL}(\mathbf{P})$ (or if $S$ is a set of terms) we let $\operatorname{Id}_{\mathbf{P}}(S)$ be the ideal of $\mathbf{P}$ generated by $\bigcup_{s \in S} \operatorname{Id}_{\mathbf{P}}(s)$. Filters in $\mathbf{P}$ and Fil $_{\mathbf{P}}(t)$ and Fil $_{\mathbf{P}}(S)$ are defined dually.

Theorem 1 (Dean [3]). Let $s$ and $t$ be terms in the variables $P$. Then $s \leq t$ holds in FL(P) if and only if one of the following holds:

(1) $s \in P$ and $t \in P$ and $s \leq t$ in $\langle P, \leq\rangle$;

(2) $s=s_{1} \vee \cdots \vee s_{n}$ and $\forall i \quad s_{i} \leq t$;

(3) $t=t_{1} \wedge \cdots \wedge t_{n}$ and $\forall i \quad s \leq t_{i}$;

(4) $s \in P$ and $t=t_{1} \vee \cdots \vee t_{n}$ and $s \in \operatorname{Id}_{\mathbf{P}}\left(\left\{t_{1}, \ldots, t_{n}\right\}\right)$;

(5) $s=s_{1} \wedge \cdots \wedge s_{n}$ and $t \in P$ and $t \in \operatorname{Fil}_{\mathbf{P}}\left(\left\{s_{1}, \ldots, s_{n}\right\}\right)$;

(6) $s=s_{1} \wedge \cdots \wedge s_{n}$ and $t=t_{1} \vee \cdots \vee t_{n}$ and $\exists i \quad s_{i} \leq t$ or $\exists j s \leq t_{j}$ or $\exists x \in P \quad s \leq x \leq t$.

Of course when $\mathbf{P}$ is an unordered set (an antichain) of size $n$, then $\mathbf{F L}(\mathbf{P})$ $\cong \mathbf{F L}(n)$. Whitman's algorithm is the same as Dean's in cases (1), (2), and (3). If $s$ is in the generating set of a free lattice, $s \leq t_{1} \vee \cdots \vee t_{n}$ if and only if $s \leq t_{i}$, for some $i$, i.e., the generators of a free lattice are join prime. This, and the dual situation, is the main difference between Whitman's and Dean's algorithms. Notice however that (4) and (5) do represent reductions. Also notice that in case (6), if $s \leq x \leq t$ in a free lattice, then, since $x$ is join prime, $s \leq x \leq t_{i}$ for some $i$. Thus the last clause of (6) in unnecessary in the case of free lattices. The simplified condition:

$$
s=s_{1} \wedge \cdots \wedge s_{n} \leq t_{1} \vee \cdots \vee t_{m}=t \text { iff } \exists i s_{i} \leq t \text { or } \exists j s \leq t_{j}
$$

is known as Whitman's condition and is denoted (W).

Definition. If $S$ and $T$ are subsets of a lattice, we say that $S$ refines $T$ (or $S$ lower refines $T$ ) if for all $s \in S$ there is a $t \in T$ with $s \leq t$. We denote this by $S \ll T . S \gg T$ is defined dually and we say that $S$ upper refines $T$ in this case.

Lemma 2. If $x \in P$ and $x \leq t_{1} \vee \cdots \vee t_{n}$ in $\mathbf{F L}(\mathbf{P})$, then there is a set $Y \subseteq P$ such that $Y \ll\left\{t_{1}, \ldots, t_{n}\right\}$ and $x \leq \bigvee Y$ in $\mathbf{F L}(\mathbf{P})$. 
Proof. By (4) of the last theorem, the hypotheses imply that $x$ is in the ideal of $\mathbf{P}$ generated by $Y=\left\{y \in P: y \leq t_{i}\right.$ for some $\left.i\right\}$. Clearly $Y \ll\left\{t_{1}, \ldots, t_{n}\right\}$. The join of $Y$ may not be defined in $\mathbf{P}$, but it is easy to see that every element of the ideal of $\mathbf{P}$ generated by $Y, \operatorname{Id}_{\mathbf{P}}(Y)$, is below $\bigvee Y$, and hence $x \leq$ $\vee Y$.

\section{CANONICAL FORM}

Each element in a free lattice has a shortest term representing it which is unique up to commutativity and associativity. This is called the canonical form of the element. This syntactical concept is closely related to arithmetic of the lattice. We will see that the elements of $\mathbf{F L}(\mathbf{P})$ also have a canonical form and that there is a nice connection between this form and the arithmetic of the lattice. A related but different canonical form is considered in [8]. Our canonical form has the nice property that when applied to free lattices, it agrees with Whitman's.

As we mentioned above the major difference between Dean's algorithm and Whitman's lies in conditions (4) and (5). However if we are dealing with a certain kind of term, which we will call adequate, these difficult conditions can be replaced with the simple free lattice conditions.

Definition. Let $\mathbf{P}$ be a finite partial lattice. A term $t$ with variables from $P$ is called adequate if it is an element of $P$ or if $t=t_{1} \vee \cdots \vee t_{n}$ is a formal join, each $t_{i}$ is adequate, and if $p \leq t$ for $p \in P$ then $p \leq t_{i}$ for some $i$. If $t$ is formally a meet the dual condition must hold.

Lemma 3. Let $s$ and $t$ be adequate terms. Then $s \leq t$ in $\mathbf{F L}(\mathbf{P})$ if and only if $s \leq t$ in $\mathbf{F L}(\langle P, \leq\rangle)$.

Proof. Of course, $\langle P, \leq\rangle$ denotes the $P$ as a partially ordered set. The lemma follows from Theorem 1 and the remarks which follow it.

An easy inductive argument shows that for every element $w$ of $\mathbf{F L}(\mathbf{P})$ there is an adequate term representing $w$. Also every term is adequate in the case of free lattices. The next theorem will show that there is a shortest adequate term representing $w$ and that this term is unique up to commutativity. We call such a term the canonical form of $w$.

Theorem 4. For each element of $\mathbf{F L}(\mathbf{P})$ there is an adequate term of minimal rank representing it, and this term is unique up to commutativity.

Proof. Suppose that $s$ and $t$ are both shortest adequate terms that represent the same element $w$ in $\mathbf{F L}(\mathbf{P})$. If either $s$ or $t$ is in $P$, then clearly $s=t$.

Observe that if $t=t_{1} \vee \cdots \vee t_{n}$ and some $t_{i}$ is formally a join, we could lower the rank of $t$ by removing the parentheses around $t_{i}$. Since $t_{i}$ is adequate, the resulting term would still adequately represent $w$. But this would violate the minimally of $t$. Thus we conclude that each $t_{i}$ is not formally a join. 
Suppose that $t=t_{1} \vee \cdots \vee t_{n}$ and $s=s_{1} \vee \cdots \vee s_{m}$. Then $t_{i} \leq s_{1} \vee \cdots \vee s_{m}$. This implies that either $t_{i} \leq s_{j}$ for some $j$, or $t_{i}=\bigwedge t_{i j}$ and $t_{i, j} \leq s$ for some $j$, or there is an $x \in P$ with $t_{i} \leq x \leq s_{1} \vee \cdots \vee s_{m}$. In the second case we have $t_{i} \leq t_{i, j} \leq t$ and thus we could replace $t_{i}$ with $t_{i j}$ in $t$ producing a shorter term still representing $w$. It is easy to see that this term is still adequate, violating the minimality of the term $t$. If the third case holds then, by the adequacy of $s, x \leq s_{j}$ for some $j$. Hence in all cases there is a $j$ such that $t_{i} \leq s_{j}$. Thus $\left\{t_{1}, \ldots, t_{n}\right\} \ll\left\{s_{1}, \ldots, s_{m}\right\}$. By symmetry, $\left\{s_{1}, \ldots, s_{n}\right\} \ll$ $\left\{t_{1}, \ldots, t_{m}\right\}$. Since both are antichains (by the minimality) they represent the same set of elements of $\mathbf{F L}(\mathbf{P})$. Thus $m=n$ and after renumbering $s_{i} \approx t_{i}$. Now by induction $s_{i}$ and $t_{i}$ are the same up to commutativity.

If $t=t_{1} \vee \cdots \vee t_{n}$ and $s=s_{1} \wedge \cdots \wedge s_{m}$, then, since neither $s$ nor $t$ is in $P$, (W) implies that either $t_{i}=t$ for some $i$ or $s_{j}=s$ for some $j$, violating the minimality.

The remaining cases can be handled by duality.

The following corollary gives an effective procedure for putting a term into canonical form.

Corollary 5. A term $t=t_{1} \vee \cdots \vee t_{n}$, with $n>1$, is in canonical form if and only if

(1) each $t_{i}$ is either in $P$ or formally a meet,

(2) each $t_{i}$ is in canonical form,

(3) $t_{i} \nless t_{j}$ for all $i \neq j$ (the $t_{i}$ 's form an antichain),

(4) if $t_{i}=\bigwedge t_{i, j}$ then $t_{i, j} \nless t$ for all $j$,

(5) $t$ is adequate.

Proof. All of these conditions are clearly necessary. For the converse we need to show that if $t$ satisfies (1)-(5) then it has minimal rank among the adequate terms which represent the same element of $\mathbf{F L}(\mathbf{P})$ as $t$. Suppose that $s=$ $s_{1} \vee \cdots \vee s_{n}$ is an adequate term of minimal rank representing the same element of $\mathbf{F L}(\mathbf{P})$ as $t$. Now using (1)-(5) and the arguments of the last theorem we get that

$$
\begin{aligned}
& \left\{t_{1}, \ldots, t_{n}\right\} \ll\left\{s_{1}, \ldots, s_{m}\right\}, \\
& \left\{s_{1}, \ldots, s_{n}\right\} \ll\left\{t_{1}, \ldots, t_{m}\right\} .
\end{aligned}
$$

Since both are antichains, we have that $n=m$ and after renumbering $s_{i} \approx t_{i}$, $i=1, \ldots, n$. The proof can now easily be completed with the aid of induction.

In free lattices the canonical form is associated with nonrefinable join representations, which, in free lattices, are unique. The next theorem will show that in a finitely presented lattice each element can have only finitely many nonrefinable join representations and these can be easily found from the canonical form. 
We define the canonical join representation of $w \in \mathbf{F L}(\mathbf{P})$ to be $w_{1} \vee \cdots \vee w_{n}$ if the canonical form of $w$ is $t_{1} \vee \cdots \vee t_{n}$ and the interpretation of $t_{i}$ in $\mathbf{F L}(\mathbf{P})$ is $w_{i}$. It is useful to separate out the elements of $P$ in such a representation. Thus let

$$
\begin{aligned}
w & =w_{1} \vee \cdots \vee w_{n} \vee x_{1} \vee \cdots \vee x_{k} \\
& =\bigvee_{i} \bigwedge_{j} w_{i, j} \vee \bigvee_{i} x_{i}
\end{aligned}
$$

be the canonical join representation of $w$ where $x_{i} \in P, i=1, \ldots, k$, and the canonical meet representation of $w_{i}$ is $w_{i}=\bigwedge w_{i, j}$.

Theorem 6. Let the canonical join representation for $w$ be given by (1). If $w=v_{1} \vee \cdots \vee v_{m}$ in $\mathbf{F L}(\mathbf{P})$ then there exists $y_{1}, \ldots y_{r} \in P$, such that

$$
w=w_{1} \vee \cdots \vee w_{n} \vee y_{1} \vee \cdots \vee y_{r}
$$

and

$$
\left\{w_{1}, \ldots, w_{n}, y_{1}, \ldots, y_{r}\right\} \ll\left\{v_{1}, \ldots, v_{m}\right\}
$$

Every nonrefinable join representation of $w$ contains $\left\{w_{1}, \ldots, w_{n}\right\}$ and also contains every $x_{i}$ which is join irreducible.

Proof. Since, for $i=1, \ldots, n$,

$$
w_{i} \leq v_{1} \vee \cdots \vee v_{m}=w
$$

we have that either (i) $w_{i} \leq v_{j}$ for some $j$, (ii) $w_{i, j} \leq w$, or (iii) $w_{i} \leq x \leq$ $w$ for some $x \in P$. If either (ii) or (iii) held, we could produce a shorter adequate term representing $w$, violating the minimality of the representation $w=w_{1} \vee \cdots \vee w_{n} \vee x_{1} \vee \cdots \vee x_{k}$. Hence (i) must hold.

Since $x_{i} \leq v_{1} \vee \cdots \vee v_{m}$, by Lemma 2 there is a set $\left\{z_{1}, \ldots, z_{s}\right\} \subseteq P$ such that $x_{i} \leq z_{1} \vee \cdots \vee z_{s}$ in $\mathbf{F L}(\mathbf{P})$, and

$$
\left\{z_{1}, \ldots, z_{s}\right\} \ll\left\{v_{1}, \ldots, v_{m}\right\} .
$$

Hence if we let $\left\{y_{1}, \ldots y_{r}\right\}$ be the union of the $z$ 's obtained from all of the $x_{i}$ 's,

$$
w=w_{1} \vee \cdots \vee w_{n} \vee y_{1} \vee \cdots \vee y_{r}
$$

and

$$
\left\{w_{1}, \ldots, w_{n}, y_{1}, \ldots, y_{r}\right\} \ll\left\{v_{1}, \ldots, v_{m}\right\}
$$

This proves everything except the statement about the join irreducible $x_{i}$ 's. First we claim that each $x_{i}$ in (1) is a maximal element of $P$ in the ideal $(w / 0) \cap P$ of $\mathbf{P}$. If $x_{i}<y \leq w$ then we could replace $x_{i}$ by $y$ in (1). The resulting expression would still correspond to an adequate term, in violation of the uniqueness of the canonical form. Now, by Theorem $1, x_{i} \leq v_{1} \vee \cdots \vee v_{m}$ means that $x_{i}$ is the ideal of $\mathbf{P}$ generated by $\bigcup\left(v_{j} / 0\right) \cap P$. This ideal is obtained from this union by alternately taking joins of subsets of this union that exist in $P$ and taking all elements less than something in the set. Obviously all such elements will be contained in $w$. But since $x_{i}$ is a maximal element in $\mathbf{P}$ 
below $w$, the only way for a join of elements of $\mathbf{P}$ below $w$ to contain $x_{i}$ is for it to equal $x_{i}$. Thus, in the case that $x_{i}$ is join irreducible, we must have $x_{i} \leq v_{j}$ for some $j$. This proves the last statement.

Notice that this proof shows that every nonrefinable join representation of $w$ refines the canonical join representation. It also shows that for $1 \leq j \leq k$ and for any nonrefinable join representation $w=\bigvee T$,

$$
x_{j}=\bigvee\left\{t \in T: t \leq x_{j}\right\} .
$$

This implies that the nonrefinable join representations of $w$ can be found from the canonical join representation of $w$ simply by replacing each join reducible $x_{j}$ with one of its nonrefinable join representations.

\section{Covers}

In [7] J. B. Nation and the author gave an effective proceedure for determining if an element of a free lattice had a lower cover, and finding it if it did. We will prove a similar result for finitely presented lattices.

Definition. A join cover of $x \in L$, where $\mathbf{L}$ is a (partial) lattice, is a finite set $S \subseteq L$ such that $x \leq \bigvee S$. We use the term meet cover for the dual notion, although this terminology is less than ideal. A subset $S$ of the join irreducible elements of $L$ is said to be closed if for every $u \in S$, every join cover of $u$ can be refined to a join cover of $u$ consisting of elements of $S$. We call a join cover $S$ of $x$ a nonrefinable join cover of $x$ if whenever $T$ is a join cover of $x$ and $T \ll S$ then $S \subseteq T$. A join cover $S$ of $x$ is nontrivial if there is no $s \in S$ with $x \leq s$.

Lemma 7. Every join irreducible element $w \in \mathbf{F L}(\mathbf{P})$ is contained in a finite closed set.

Proof. Define a set $\mathbf{J}^{\prime}(w)$ of join irreducibles associated with $w$ as follows. If $w \in P$ then $\mathbf{J}^{\prime}(w)=\varnothing$. If the canonical meet representation of $w$ is given by

$$
w=\bigwedge_{i} \bigvee_{j} w_{i, j} \wedge \bigwedge_{k} x_{k}
$$

then we let

$$
\mathbf{J}^{\prime}(w)=\{w\} \cup \bigcup_{i, j} \mathbf{J}^{\prime}\left(w_{i, j}\right)
$$

We claim that if we let $\mathrm{T}(w)$ be the union of $\mathrm{J}^{\prime}(w)$ and the join irreducible elements of $P, \mathrm{~T}(w)$ is closed. If $w \in P$ then this follows from Lemma 2. Clearly if $v \in \mathrm{T}(w)$ then $\mathrm{T}(v) \subseteq \mathrm{T}(w)$. Hence it suffices to show that any join cover of $w$ refines to one in $\mathrm{T}(w)$. Suppose $w \leq \bigvee U$. Since $w \in \mathrm{T}(w)$, the claim is obvious if $w \leq u$ for some $u \in U$. Otherwise, $w_{i} \leq \bigvee U$, for some $i$, or $x_{j} \leq \bigvee U$, for some $j$. In the latter case the claim follows from Lemma 2 again. It the former case $w_{i, j} \leq \bigvee U$ for all $j$. Since the $\mathrm{T}\left(w_{i, j}\right) \subseteq \mathrm{T}(w)$, an induction argument shows that there is a refinement of $U$ to a join cover 
$V_{i, j}$ of $w_{i, j}$. If $V=\bigcup_{j} V_{i, j}$, then $V$ is a join cover of $w_{i}$, and hence $w$, refining $U$.

Lemma 8. The intersection of a finite closed set with an arbitrary closed set is closed.

Proof. Suppose that both $S$ and $T$ are closed sets with $S$ finite and let $w \in$ $S \cap T$. If $w \leq \bigvee U$ then there is a join cover $V \subseteq S$ refining $U$. Moreover, since $S$ is finite, we may assume that if $V^{\prime} \subseteq S$ is a join cover refining $V$, then $V \subseteq V^{\prime}$. Since $T$ is also closed, it has a subset $V_{1}$ with $V_{1} \ll V$ which is a join cover of $w$. But since $S$ is closed, it has a subset $V_{2} \ll V_{1}$ which is a join cover of $w$. By the choice of $V$, we have

$$
V \subseteq V_{2} \ll V_{1} \ll V \text {. }
$$

This implies $V=V_{1}$. Thus $V \subseteq S \cap T$ showing that $S \cap T$ is closed.

Using the last two lemmas we can show that if $w$ is a join irreducible element of $\mathbf{F L}(\mathbf{P})$ then there is a unique smallest closed set containing $w$, which is denoted $\mathrm{J}(w)$. In fact an induction argument shows that a finite closed set of minimum cardinality containing $w$ will be $\mathrm{J}(w)$. The lemmas also show that $\mathrm{J}(w)$ can be characterized as the smallest set $S$ containing $w$ such that if $u \in S$ and $V$ is a nonrefinable join cover of $u$, then $V \subseteq S$. We extend the definition of $\mathrm{J}(w)$ to include all elements of $\mathbf{F L}(\mathbf{P})$ by defining $\mathrm{J}(w)=\bigcup_{u} \mathrm{~J}(u)$, where the union is over all elements $u$ which lie in a nonrefinable join representation of $w$. Notice that all the elements of $\mathrm{J}(w)$ are join irreducible. A argument similar to the proof of the last two lemmas shows that this extended $\mathrm{J}(w)$ is the smallest closed set such that any join cover of $w$ can be refined to one contained in $\mathrm{J}(w)$.

For $w \in \mathbf{F L}(\mathbf{P})$, we define the rank of $w$ to be the rank of the canonical form of $w$. In the proof of Lemma 7, a closed set containing $w$ was constructed as the union of $\mathbf{J}^{\prime}(w)$ and a subset of $P$. Since the elements of $\mathbf{J}^{\prime}(w)-\{w\}$ all have rank less than the rank of $w$, we have the following theorem.

Theorem 9. For each $w \in \mathbf{F L}(\mathbf{P})$ there is a unique smallest closed set, $\mathbf{J}(w)$, with the property that every join cover of $w$ can be refined to one whose elements all lie in $\mathrm{J}(w)$. If $w \notin P$ then every element of $\mathrm{J}(w)-\{w\}$ has lower rank than $w$. If $w \in P$ then $\mathrm{J}(w) \subseteq P$.

Let $\mathbf{P}^{\wedge}$ be the closure under meets of $P$ in $\mathbf{F L}(\mathbf{P})$. An element with no nontrivial join cover is said to be join prime.

Corollary 10. If $w \in \mathbf{F L}(\mathbf{P})$ then $\mathrm{J}(w) \cap \mathbf{P}^{\wedge} \neq \varnothing$. Furthermore, if $w \in P^{\wedge}$ then $\mathrm{J}(w) \cap P \neq \varnothing$ unless $w$ is join prime.

Proof. We prove this by induction on the rank of $w$. If $w \in P$ then this corollary follows from the previous theorem. Thus we may assume $w \notin P$. If $w$ is not join irreducible then $\mathrm{J}(w)$ is nonempty and all of its elements have 
rank lower than the rank of $w$, so the result follows easily by induction. Thus assume that $w$ is join irreducible and that $w \notin \mathbf{P}^{\wedge}$. If $v \in \mathrm{J}(w)-\{w\}$ then by the last theorem the rank of $v$ is less that of $w$. By induction $\mathrm{J}(v) \cap \mathbf{P}^{\wedge} \neq \varnothing$. Since $\mathrm{J}(v) \subseteq \mathrm{J}(w)$, we are done unless $\mathrm{J}(w)=\{w\}$. However, if this were the case then $w$ would be join prime. Since $w$ is not in $\mathbf{P}^{\wedge}$ then one of the canonical meetands, say $w_{1}$, of $w$ is not in $P$. If the canonical joinands of $w_{1}$ are $w_{1, j}, j=1, \ldots, n$, then, by (4) of Corollary $5,\left\{w_{1,1}, \ldots, w_{1, n}\right\}$ is a nontrivial join cover of $w$. Thus $w$ is not join prime. If $w \in P^{\wedge}-P$ and is not join prime, then by the last theorem $\mathrm{J}(w)-\{w\} \subseteq P$. Since $w$ is not join prime, this set is not empty.

Definition. $\mathbf{L}(w)$ is the join closure of $\mathbf{J}(w)$ in $\mathbf{F L}(\mathbf{P})$ with a new least element adjoined. In symbols

$$
\mathbf{L}(w)=\mathbf{J}(w)^{\vee} \cup\{0\} .
$$

Since $\mathbf{L}(w)$ is a finite, join closed subset of $\mathbf{F L}(\mathbf{P})$ with a least element adjoined, it is a lattice. Also note that joins in $\mathbf{L}(w)$ agree with those in FL( $\mathbf{P}$ ) .

Define a map $f: \mathbf{F L}(\mathbf{P}) \rightarrow \mathbf{L}(w)$ by

$$
f(u)=\bigvee\{v \in \mathbf{J}(w): v \leq u\} .
$$

This map played an important role in [7] and will also be important here. We refer to it as the standard epimorphism or the standard homomorphism. The next theorem shows that it is a homomorphism.

Theorem 11. The standard homomorphism is a homomorphism.

Proof. Clearly $f$ preserves order and satisfies $f(u) \leq u$. Hence, for $u \in$ $\mathbf{F L}(\mathbf{P})$ and $v \in \mathbf{J}(w), v \leq u$ if and only if $v \leq f(u)$. The reader can check that $f$ preserves meets. For joins, suppose that $a, b \in \mathbf{F L}(\mathbf{P})$. Clearly $f(a \vee b) \geq f(a) \vee f(b)$. For the other direction, suppose that $v \leq a \vee b$, for some $v \in \mathbf{J}(w)$. Since $v \in \mathbf{J}(w)$, there is a set $T \subseteq \mathrm{J}(w)$ with

$$
T \ll\{a, b\}, \quad v \leq \bigvee T .
$$

Since $T \subseteq \mathrm{J}(w), f(t)=t$, for $t \in T$. Thus

$$
v \leq \bigvee T=\bigvee_{t \in T} f(t) \leq f(a) \vee f(b),
$$

showing that $f(a \vee b)=f(a) \vee f(b)$.

We are interested in determining which elements of $\mathbf{F L}(\mathbf{P})$ have lower covers. Notice that a join irreducible element $w$ has a lower cover if and only if it is completely join irreducible, that is, there is a greatest element, always denoted $w_{*}$, strictly less than $w$. We let $\kappa(w)$ denote the set of elements $v$ maximal with the property that

$$
v \geq w_{*}, \quad v \nsupseteq w .
$$


Theorem 12. Let $w \in \mathbf{F L}(\mathbf{P})$ be completely join irreducible. Then $\kappa(w)$ is finite and if $v$ satisfies (3), then $v \leq m$ for some $m \in \kappa(w)$. Moreover, either $|\kappa(w)|=1$ or $\kappa(w) \subseteq P$.

Proof. We will show that $\kappa(w)$ is the set of members of maximal (i.e., nonrefinable) meet representations of $w_{*}$, which are not above $w$. By the dual of Theorem 6 this set consists of the meet irreducible elements of the canonical meet representation of $w_{*}$, which are not above $w$, together with those elements $p \in P$ maximal with the property $p \geq w_{*}$ but $p \nsucceq w$. If $v \geq w_{*}$ but not $v \nsucceq w$, then

$$
w_{*}=v \wedge w .
$$

By the dual of Theorem 6 , this meet can be refined to a maximal one, proving the first part of the theorem.

Suppose that $\kappa(w)$ contains an element $m$ not in $P$. Now if $w_{*}=v \wedge w$ then by the dual of Theorem 6 this meet representation can be upper refined to a nonrefinable one. Again by Theorem 6 this refinement must contain $m$. Since $m \nsucceq w$ we must have $m \geq v$. This clearly implies $|\kappa(w)|=1$.

The next theorem shows that the question of the existence of a lower cover of an arbitrary element can be reduced to the question for join irreducible elements.

Theorem 13. An element $w \in \mathbf{F L}(\mathbf{P})$ has a lower cover if and only if there is a completely join irreducible element in some nonrefinable join representation of $w$.

Proof. Let $u$ be a completely join irreducible element in a nonrefinable join representation of $w$. Let $v$ be the join of the other elements of this representation. Then $u_{*} \vee v<w$ and hence

$$
u_{*}=u \wedge\left(u_{*} \vee v\right)
$$

Thus there is an $m \in \kappa(u)$ with $m \geq u_{*} \vee v$. Since $\kappa(u)$ is finite, we can choose an $m \in \kappa(u)$ such that $m \wedge w$ is a maximal element in the set

$$
\{n \wedge w: n \in \kappa(u), n \geq v\} .
$$

Then it is easy to check that $w \succ w \wedge m$.

Conversely, suppose that $w \succ v$ for some $v$. By Theorem 6 there are only finitely many elements involved in nonrefinable join representations of $w$. So we can choose $u$ to be a minimal element of a nonrefinable join representation with the properties $u \npreceq v$. If

$$
u \wedge v<a<u
$$

then $a \vee v=w$. By Theorem 6 this join can be refined to a nonrefinable join, $w=\bigvee T$. There must be a $t \in T$ with $t \not v$. Hence $t \leq a<u$, contradicting the minimality of $u$. Thus $u \succ u \wedge v$, and so $u$ is completely join irreducible. 
An epimorphism $f: \mathbf{K} \rightarrow \mathbf{L}$ is called lower bounded if each element $x \in L$ has a least preimage. This least preimage, when it exists, is denoted $\beta(x)$. Upper bounded is defined dually and the greatest preimage, when it exists is denoted $\alpha(x) . f$ is bounded if it is both upper and lower bounded. Notice that if follows immediately from the definition that the standard homomorphism is lower bounded.

Theorem 14. A join irreducible element $w \in \mathbf{F L}(\mathbf{P})$ is completely join irreducible if and only if the standard homomorphism $f: \mathbf{F L}(\mathbf{P}) \rightarrow \mathbf{L}(w)$ is bounded.

Proof. First suppose that $f$ is bounded. Clearly $w$ is join irreducible in $\mathbf{L}(w)$. Let $w_{+}$be its lower cover in $\mathbf{L}(w)$, that is,

$$
w_{\dagger}=\bigvee\{v \in \mathbf{J}(w): v<w\}
$$

Also define $\kappa_{\mathbf{L}(w)}(w)$ to be the set of all meet irreducibles $m \in \mathbf{L}(w)$ satisfying $m \geq w_{\dagger}, m \nsucceq w$. It is easy to check that $w \succ w \wedge \alpha(m)$ for $m \in \kappa_{\mathbf{L}(w)}(w)$.

Conversely, suppose that $w$ is completely join irreducible with lower cover $w_{*}$. Let $\mathbf{L}=\mathbf{F L}(\mathbf{P}) / \psi\left(w, w_{*}\right)$, where $\psi\left(w, w_{*}\right)$ is the unique largest congruence separating $w$ from $w_{*}$ (such a congruence exists by Dilworth's characterization of lattice congruences, see [1]). Now $\mathbf{L}(w)$ is an image of $\mathbf{F L}(\mathbf{P})$ separating $w$ and $w_{*}$. It is not difficult to show that any image of $\mathbf{L}(w)$ must identify $w$ and $w_{\dagger}$ (see Theorem 4.1 of [7]). Thus $\mathbf{L}(w)=\mathbf{L}$. Recall that $\mathbf{P}^{\wedge}$ is the meet closure of $\mathbf{P}$ in $\mathbf{F L}(\mathbf{P})$. Consider $\mathbf{P}^{\wedge(\vee \wedge)^{n}}$, the $n$-fold closure of $\mathbf{P}^{\wedge}$ under joins and meets. This is a finite subset of $\mathbf{F L}(\mathbf{P})$ closed under meets and possessing a greatest element, hence a lattice. If $n$ is large enough this lattice will satisfy the relations of $\mathbf{P}$ and the proof of Theorem 11 shows that the (dual) standard epimorphism

$$
g: \mathbf{F L}(\mathbf{P}) \rightarrow \mathbf{P}^{\wedge(\vee \wedge)^{n}}
$$

is a homomorphism and it is upper bounded. If we choose $n$ large enough, $g$ will separate $w$ and $w_{*}$. Thus there will be an epimorphism $h: \mathbf{P}^{\wedge(\vee \wedge)^{n}} \rightarrow \mathbf{L}$. If $f$ is the natural map from $\mathbf{F L}(\mathbf{P})$ to $\mathbf{L}$ then $f=h g$. Since $\mathbf{P}^{\wedge(\vee \wedge)^{n}}$ is finite, $h$ is clearly bounded. Since $g$ is upper bounded, $f$ is upper bounded. Since $f$ is the standard homomorphism, it is also lower bounded.

In the next section we shall show that there is an effective algorithm to decide if an element in a finitely presented lattice has a lower cover. We close this section with a theorem which gives strong necessary conditions for a join irreducible element to have a lower cover. The definition of $w_{\dagger}$ is given in (4). Let $\mathrm{K}(w)$ be the set of maximal elements of the set

$$
\left\{v \in \mathbf{L}(w): w_{\dagger} \leq v, w \not \leq v\right\} .
$$

Let $S$ denote the maximal elements of the set

$$
\left\{p \in P: w_{\dagger} \leq p, w \leq p\right\} \text {. }
$$


Theorem 15. The following are necessary conditions for a join irreducible element $w \in \mathbf{F L}(\mathbf{P})$ to be completely join irreducible:

(1) each element $v \in \mathrm{J}(w)$ is completely join irreducible;

(2) if $w \leq \vee \mathrm{K}(w)$ then for each $u \in \mathrm{L}(w)$ with $w \mathbb{z} w_{\dagger} \vee u$, there is a $p \in S$ such that $w_{+} \vee u \leq p$

(3) if $w \leq \bigvee \mathrm{K}(w)$ then $w \wedge s_{1}=w \wedge s_{2}$ for all $s_{1}, s_{2} \in S$ (the meets are calculated in $\mathbf{F L}(\mathbf{P}))$;

(4) if $w \leq \bigvee \mathrm{K}(w)$ then for all $s_{1}, s_{2} \in S, s_{1} \vee\left(s_{2} \wedge\left(s_{1} \vee w\right)\right)$ equals $s_{1}$ or $s_{1} \vee w$.

Proof. Assume that $w$ is completely join irreducible. Let $v \in \mathrm{J}(w)$. Then $\mathbf{J}(v) \subseteq \mathbf{J}(w)$. Let $f: \mathbf{F L}(\mathbf{P}) \rightarrow \mathbf{L}(w)$ and $g: \mathbf{F L}(\mathbf{P}) \rightarrow \mathbf{L}(v)$ be the standard epimorphisms. Just as in the proof of Theorem 11 , we can show that there is a epimorphism $h: \mathbf{L}(w) \rightarrow \mathbf{L}(v)$ such that $g=h f$. By Theorem 14, $f$ is bounded. Since $h$ is obviously bounded, it follows that $g$ is bounded. Thus $v$ is completely join irreducible again by Theorem 14 .

If $|\kappa(w)|=1$ and $m$ is the unique element, then $f(m)$ contains all elements of $\mathbf{L}(w)$ which are above $w_{\dagger}$ but not above $w$, where $f$ is the standard homomorphism from $\mathbf{F L}(\mathbf{P})$ onto $\mathbf{L}(w)$. In this case $w \notin \mathbb{Z}(w)$. Thus for parts (2), (3), and (4) we may assume that $|\kappa(w)|>1$. This implies that $\kappa(w) \subseteq P$ by Theorem 12. Now if $u \in \mathrm{L}(w)$ satisfies $w \leq w_{\dagger} \vee u$ then there is an element $v \in \mathrm{K}(w)$ with $w_{\dagger} \vee u \leq v$. Since the standard homomorphism is upper bounded, $\alpha(v) \in \kappa(w) \subseteq P$, completing the proof of (2).

For (3), by using this same reasoning as above, we see that $S$ is the set of maximal elements of the set

$$
\left\{p \in P: w_{*} \leq p, w \leq p\right\} .
$$

Thus $s \wedge w=w_{*}$ for each $s \in S$. Moreover, it follows that, for $s \in S, s$ is completely meet irreducible with unique upper cover $s \vee w$. (4) follows easily from this.

\section{BOUNDED HOMOMORPHISMS}

Theorem 14 shows the importance of bounded homomorphisms. In this section we will give an effective procedure for determining if a homomorphism from $\mathbf{F L}(\mathbf{P})$ to a finite lattice is bounded. McKenzie [9] has given such an algorithm for finitely generated free lattices.

Let

$$
f: \mathbf{F L}(\mathbf{P}) \rightarrow \mathbf{L}
$$

be an epimorphism onto a finite lattice $\mathbf{L}$. For $a \in L$ we let $\beta(a)$ denote the least preimage of $a$ under $f$ if it exists. McKenzie's procedure found a descending sequence $\beta_{0}(a) \geq \beta_{1}(a) \geq \cdots$ of approximations of $\beta(a)$, called the (lower) limit table. This sequence was always dually cofinal in $f^{-1}(a)$. Thus, if $\beta(a)$ exists, it must equal $\beta_{n}(a)$ for some $n$. What turns out to be a 
closely related approach was initiated by Jónsson in his unpublished notes, see [9]. We give a rough sketch of Jónsson's ideas. If $a$ and $b$ are join irreducible elements of a finite lattice $\mathbf{L}, a$ is said to depend on $b$ if $b$ is a member of a nontrivial, nonrefinable join cover of $a$. Then $\mathbf{L}$ is a bounded homomorphic image of a free lattice if and only if this dependency relation contains no cycle. Of course every finite lattice is finitely presented and the identity map is a bounded homomorphism. Thus bounded images of finitely presented lattices can have cycles. Very roughly we can say that the epimorphism (5) is lower bounded if the cycles occurring in $\mathbf{L}$ already occur in $\mathbf{P}$. Our algorithm combines ideas from both McKenzie's and Jónsson's approach, but is more complicated than either.

Notation. For $a \in \mathbf{L}$, a finite lattice, we let $\mathrm{JC}_{\mathbf{L}}(a)$ (or $\mathrm{JC}(a)$ if the context is clear) be the set of all nonrefinable, nontrivial join covers of $a$. For $a \in \mathbf{J}(\mathbf{L})$ (where $\mathbf{J}(\mathbf{L})$ denotes the join irreducible elements of $\mathbf{L}$ ) let $\mathbf{J}_{\mathbf{L}}(a)$ (or $\mathbf{J}(a)$ ) be the smallest subset of $\mathbf{J}(\mathbf{L})$ containing $a$ and such that if $b \in \mathbf{J}_{\mathbf{L}}(a)$ then $\cup \mathrm{JC}(b) \subseteq \mathbf{J}_{\mathbf{L}}(a)$. For an epimorphism $f: \mathbf{F L}(\mathbf{P}) \rightarrow \mathbf{L}$, where $\mathbf{L}$ is finite, let $D_{-1}=\varnothing$ and let $\beta_{0}: \mathbf{L} \rightarrow \mathbf{F L}(\mathbf{P})$ be a map which satisfies

(1) $f \beta_{0}(a)=a$, for all $a \in L$,

(2) $\beta_{0}$ preserves order,

(3) $\beta_{0}(f(p)) \leq p$ for all $p \in P$.

Now define $D_{n} \subseteq \mathrm{J}(\mathbf{L})$ and $\beta_{n}: \mathbf{J}(\mathbf{L}) \rightarrow \mathbf{F L}(\mathbf{P})$ inductively as follows.

$$
\begin{gathered}
D_{n}=\left\{a \in \mathrm{J}(\mathbf{L}): \forall b \in \mathrm{J}(a), \forall S \in \mathrm{JC}(b), \beta_{n}(b) \leq \bigvee \beta_{n}(S)\right\}, \\
\beta_{n+1}(a)=\beta_{n}(a) \wedge \bigwedge_{\substack{S \in \mathrm{JC}(a) \\
S \subseteq D_{n}}} \bigvee \beta_{n}(S)
\end{gathered}
$$

\section{Lemma 16.}

(1) $\beta_{n+1}(a) \leq \beta_{n}(a)$,

(2) if $a \in D_{n}$ then $\beta_{n}(a)=\beta(a)$,

(3) $D_{n} \subseteq D_{n+1}$,

(4) $\beta_{n+1}(a)=\beta_{0}(a) \wedge \bigwedge\left\{\bigvee \beta_{n}(S): S \in \mathrm{JC}(a)\right.$ and $\left.S \subseteq D_{n}\right\}$,

(5) if $D_{n}=D_{n+1}$ then $D_{n}=D_{n+k}, k \geq 0$.

Proof. (1) is obvious. For (2) first note that if $a \in D_{n}$ then $\beta_{n}(a)=\beta_{n+1}(a)$ by the definition of $D_{n}$. To prove that this is $\beta(a)$ we show by induction on the rank of $w \in \mathbf{F L}(\mathbf{P})$ that

$$
f(w) \geq a \text { implies } w \geq \beta_{n}(a) .
$$

Of course the fact that $\beta(a)=\beta_{n}(a)$ follows from this. If $w \in P$ then this follows from the defining properties of $\beta_{0}$ and part (1) of this lemma. The argument is straighforward if $w$ is the meet of simpler terms, so assume that $w=w_{1} \vee \cdots \vee w_{k}$. Then $\bigvee f\left(w_{i}\right)=f(w) \geq a$. If $f\left(w_{i}\right) \geq a$ for some $i$, the argument is again easy. Thus we assume that $\left\{f\left(w_{i}\right): i=1, \ldots, k\right\}$ is 
a nontrivial join cover of $a$. Hence there is an $S \in \mathrm{JC}(a)$ such that $S \ll$ $\left\{f\left(w_{i}\right): i=1, \ldots, k\right\}$, i.e., for each $s \in S$, we have $s \leq f\left(w_{i}\right)$, for some $i$. Since $a \in D_{n}$ we have that $S \subseteq D_{n}$. Since the $w_{i}$ 's are of lower rank than $w$, for each $s \in S$, there is an $i$ such that $w_{i} \geq \beta_{n}(s)$. Hence

$$
\beta_{n}(a)=\beta_{n+1}(a) \leq \bigvee \beta_{n}(S) \leq \bigvee w_{i}=w
$$

Of course (3) follows easily from this. In (4) we are trying to show that in the definition of $\beta_{n+1}(a)$ the $\beta_{n}(a)$ term can be replaced with $\beta_{0}(a)$. This problem easily reduces to showing that

$$
\beta_{0}(a) \wedge \bigwedge_{\substack{S \in \mathrm{JC}(a) \\ S \subseteq D_{n}}} \bigvee \beta_{n}(S) \leq \beta_{n}(a)
$$

By induction

$$
\beta_{n}(a)=\beta_{0}(a) \wedge \bigwedge_{\substack{S \in \mathrm{JC}(a) \\ S \subseteq D_{n-1}}} \bigvee \beta_{n-1}(S)
$$

Now (8) follows from (1) and (3).

Using the parts we have proved so far we have that

$$
\beta_{n+1}(a)=\beta_{0}(a) \wedge \bigwedge_{\substack{S \in \mathrm{JC}(a) \\ S \subseteq D_{n}}} \bigvee \beta(S)
$$

From this formula it follows that if $D_{n}=D_{n+1}$ then $\beta_{n+1}=\beta_{n+2}$. This in turn implies that $D_{n+1}=D_{n+2}$, from which (5) follows.

Theorem 17. Let $f: \mathbf{F L}(\mathbf{P}) \rightarrow \mathbf{L}$ be an epimorphism onto a finite lattice $\mathbf{L}$. Let $\beta_{0}: \mathbf{L} \rightarrow \mathbf{F L}(\mathbf{P})$ be a map which satisfies (1)-(3) of the definition of $\beta_{0}$ and let $\beta_{n}$ and $D_{n}$ be defined by (6) and (7). Then $f$ is lower bounded if and only if $D_{n}=\mathrm{J}(\mathbf{L})$ for some $n$.

Proof. If $D_{n}=\mathrm{J}(\mathbf{L})$ then, by (2) of Lemma $16, \beta$ is defined on $\mathrm{J}(\mathbf{L})$. It is easy to see that $\beta$ preserves joins and thus is defined everywhere.

Conversely suppose that $f$ is lower bounded. If $a \in \mathrm{J}(\mathbf{L})$ we will prove by induction on the rank of $\beta(a)$ that $a \in D_{n}$ for some $n$. First suppose that $\beta(a) \in P$. Then by property (3) of $\beta_{0}, \beta(a) \geq \beta_{0}(f(\beta(a)))=\beta_{0}(a)$. Hence $\beta_{0}(a)=\beta(a)$. Now if $S \in \mathrm{JC}(a)$ then clearly $\beta(a) \leq \bigvee \beta(S)$. By Lemma 2 there is a set $Y \subseteq P$ with $Y \ll \beta(S)$ and $\beta(a) \leq \bigvee Y$. Applying $f$ and using the fact that $S$ is nonrefinable, we get $S \subseteq f(Y)$. Now

$$
\beta(S) \subseteq \beta(f(Y)) \ll Y \ll \beta(S) .
$$

Since $f(\beta(S))=S, \beta(S)$ is an antichain. Hence $Y=\beta(S)$. Thus it follows that if $\beta(a) \in P$ then $\beta(b) \in P$ for all $b \in \mathrm{J}(a)$, and that $\beta_{0}(b)=\beta(b)$. From this it follows that $a \in D_{0}$.

Now assume that $\beta(a) \notin P$. Let $S \in \mathrm{JC}(a)$. Then $\beta(a) \leq \bigvee \beta(S)$ is nontrivial, since otherwise $a \leq \bigvee S$ would be trivial. Now by Theorem 9 the cover $\beta(a) \leq \bigvee \beta(S)$ can be refined to a cover $U$ with elements of strictly 
lower rank than the rank of $\beta(a)$. Since $U \ll \beta(S), f(U) \ll f(\beta(S))=S$. Thus $S \subseteq f(U)$, and hence

$$
\beta(S) \subseteq \beta(f(U)) \ll U \ll \beta(S) .
$$

Thus as before $\beta(S)=U$. Thus by induction we have that for all $b \in \mathbf{J}_{\mathbf{L}}(a)-$ $\{a\}, b \in D_{n}$, for some $n$. Now using part (2) of Lemma 16 and (6) and (7), it is easy to see that $a \in D_{n+1}$.

Now we able to solve the problem of effectively finding the covers of an element of $\mathbf{F L}(\mathbf{P})$.

Corollary 18. There is an algorithm which, when given a finite partial lattice and a lattice term $t$, determines if $t^{\mathbf{F L}(\mathbf{P})}$ has any lower covers in $\mathbf{F L}(\mathbf{P})$ and finds them if it does.

Proof. Let $w=t^{\mathbf{F L}(\mathbf{P})}$. By Theorem 13 the question of lower covers of $w$ can be reduced to that of the lower covers of the join irreducible elements in the nonrefinable join covers of $w$. Moreover the proof of that theorem shows how to find all of the lower covers of $w$ from the lower covers of all the completely join irreducible elements which lie in some nonrefinable join representation of $w$. Using Theorem 6, one can effectively find elements of all nonrefinable join representations of $w$. Thus we can restrict our attention to the case $w$ is join irreducible.

So assume that $w$ is join irreducible. Looking at the proof of Lemma 7, we see that it is possible to effectively find $\mathrm{J}(w)$ and hence $\mathbf{L}(w)$. Now by Theorem 14 and the results of this section, or more accurately their duals, we see that one can effectively decide if $w$ has a lower cover. If it does have a lower cover, which would then be unique since $w$ is join irreducible, we can then find $\kappa(w)$ as follows. Let $f: \mathbf{F L}(\mathbf{P}) \rightarrow \mathbf{L}(w)$ be the standard epimorphism. By Theorem $14 f$ is bounded. Let $\alpha: \mathbf{L}(w) \rightarrow \mathbf{F L}(\mathbf{P})$ map each element to its maximal inverse image. Let $\mathrm{K}(w)$ be the set of maximal elements of the set

$$
\left\{v \in \mathbf{L}(w): v \geq w_{\dagger}, v \nsupseteq w\right\},
$$

where $w_{\dagger}$ is defined in (4). Now it is easy to check that $\kappa(w)$ is $\alpha(\mathrm{K}(w))$. Of course $w_{*}=w \wedge v$ for any $v \in \kappa(w)$.

\section{A COVERLESS FINITELY PRESENTED LATTICE}

Let $X$ be a finite, nonempty set and let $\mathbf{L}$ be the finitely presented lattice generated by $X$ subject to the relations:

$$
\bigwedge Y \leq \bigvee(X-Y)
$$

If $|X|=1$ or 2 then $\mathbf{L}$ is trivial. If $|X|=3$ then $\mathbf{L}$ is isomorphic to $\mathbf{M}_{3}$, the lattice diagrammed in Figure 1. If $|X| \geq 4$ we will show that in $L$ no element covers any other element. 


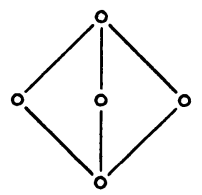

Figure 1

In order to put $\mathbf{L}$ into the form $\mathbf{F L}(\mathbf{P})$, we need to have an element $r_{Y} \in P$ to represent $\bigvee Y$, for $Y \subseteq X$ and an element $q_{Y}$ to represent the meet of $Y$. Thus we let

$$
R=\left\{r_{Y}: Y \subseteq X\right\} \text { and } Q=\left\{q_{Y}: Y \subseteq X\right\} .
$$

Let $P^{\prime}=X \cup R \cup Q \cup\{0,1\}$ and define an equivalence relation on $P^{\prime}$ by

$$
\begin{aligned}
& 1=q_{\varnothing}=r_{X}=r_{X-\{x\}}, \\
& 0=r_{\varnothing}=q_{X}=q_{X-\{x\}}, \\
& x=q_{\{x\}}=r_{\{x\}} .
\end{aligned}
$$

for $x \in X$. Let $P$ be $P^{\prime}$ with the above identification. Define an order on $P$ by

$$
\begin{array}{ll}
r_{Y} \leq r_{Z} & \text { if } Y \subseteq Z, \\
q_{Y} \leq q_{Z} & \text { if } Y \supseteq Z, \\
q_{Y} \leq r_{Z} & \text { if } Y \cup Z=X \text { or } Y \cap Z \neq \varnothing .
\end{array}
$$

Define join relations on $P$ by $r_{Y}=\bigvee Y$, for $Y \subseteq X$, and define meet relations by $q_{Y}=\wedge Y$. Let $\mathbf{P}$ be the partial lattice on $P$ defined by this order and these relations.

In order to establish that $\mathbf{F L}(\mathbf{P})$ has no covers, we will first show that if $w \in \mathbf{F L}(\mathbf{P})$ then $\mathbf{J}(w) \cap X \neq \varnothing$. If $w$ had a lower cover then by Theorem 13 and part (1) of Theorem 15, some element of $X$ would have a lower cover, which we shall show is not the case. Since 0 does not have a lower cover we will assume throughout this discussion that $w \neq 0$. By Corollary $10, \mathrm{~J}(w)$ intersects $P^{\wedge}$, so we may assume that $w \in P^{\wedge}$. We wish to show that $w$ is not join prime. Since the $q_{Y}$ 's are closed under meets, the general form of $w$ is

$$
q_{Y} \wedge r_{Z_{1}} \wedge \cdots \wedge r_{Z_{k}} \text { or } r_{Z_{1}} \wedge \cdots \wedge r_{Z_{k}}
$$

where $\left|Z_{i}\right| \geq 2$. Clearly, by the defining relations (10), $q_{Y}$ is not join prime; thus $k \geq 1$. Now $Z_{1}$ is a join cover of $w$. Suppose that this was a trivial join cover of $w$. Then $w \leq z$ for some $z \in Z_{1}$. By Theorem 1 this implies that $z \in$ Fil $_{\mathbf{P}}(w)$. Using the fact that the meets defined in $\mathbf{P}$ involve only elements of $X$, we see that the only elements of $X$ which lie in Fil $_{\mathbf{P}}(w)$ are in $Y$. But if $z \in Y$, then $q_{Y} \leq r_{Z_{1}}$, which would imply the representation (11) is redundant, which we can assume is not the case. Hence we have shown that no 
element of $P^{\wedge}$ is join prime. By Corollary 10 this implies that $\mathrm{J}(w) \cap P \neq \varnothing$ for all $w \in \mathbf{F L}(\mathbf{P})$.

To prove the stronger fact that $\mathrm{J}(w) \cap X \neq \varnothing$, consider $q_{Y}$. If $|Y|=1$ then $q_{Y} \in X$ and if $|Y| \geq|X|-1$ then $q_{Y}=0$. Hence we may assume that $2 \leq|Y|<|X|-1$. Now

$$
q_{Y} \leq \bigvee(X-Y)
$$

and we claim that this is a nonrefinable join cover of $q_{Y}$. By Theorem 9 any refinement of (12) could be further refined to elements of $P$. Thus if (12) could be properly refined, then for some $x \in X-Y$ we would have

$$
q_{Y} \leq \bigvee(X-Y-\{x\}) \vee \bigvee_{z \neq x} q_{\{x, z\}}
$$

By Theorem 1 this implies that, for this particular $x$,

$$
q_{Y} \in \operatorname{Id}_{\mathbf{P}}(X-Y-\{x\}) \cup\left\{q_{\{x, z\}}: z \neq x\right\} .
$$

However, since the joins defined in $\mathbf{P}$ only involve elements of $X$, this would imply $q_{Y} \leq \bigvee(X-Y-\{x\})$, i.e., $q_{Y} \leq r_{X-Y-\{x\}}$ in $\mathbf{P}$, which is not the case. Thus we conclude that $\mathbf{J}(w) \cap X \neq \varnothing$ for all $0 \neq w \in \mathbf{F L}(\mathbf{P})$.

As mentioned above, we now see that if any element of $\mathbf{F L}(\mathbf{P})$ had a lower cover, then some $x \in X$ would be completely join irreducible. We will show that this is not the case. Since $x \leq \bigvee(X-\{x\})$ arguments as above show that $\mathrm{J}(x)=X$. It follows that $\mathrm{L}(x)=R$. As a lattice $\mathbf{R}$ is isomorphic to the lattice of subsets of $X$ with the coatoms removed. A diagram of $\mathbf{R}$ in the case $X=\{x, y, z, t\}$ is given in Figure 2, with $r_{x, y}$ representing $r_{\{x, y\}}$, etc. Assume that $|X| \geq 4$.

In $\mathbf{R}, x_{\dagger}$ is the least element. The set $S$, defined before Theorem 15 to be the maximal elements of $\left\{p \in P: x_{\dagger} \leq p, x \notin p\right\}$, is, in the present case, $\left\{r_{X-\{x, y\}}: y \in X, y \neq x\right\}$. It is easy to check that $x \leq \bigvee \mathrm{K}(x)$ and, using Dean's Theorem, that condition (3) of Theorem 15 fails if $|X| \geq 4$. Hence $x$ is not completely join irreducible and so no element of $\mathbf{F L}(\mathbf{P})$ has a lower cover.

Consider the free lattice $\operatorname{FL}(X)$, again assuming $X$ is finite. Then

$$
\bigwedge Y \wedge \bigvee(X-Y) \prec \bigwedge Y
$$

as is well known. The above example shows that by collapsing these prime quotients we collapse all the prime quotients of $\mathbf{F L}(X)$. This fact can be derived from the work of McKenzie [9]. Namely he shows that if $\psi$ is the maximal congruence separating a prime quotient of $\mathbf{F L}(X)$, then $\mathbf{F L}(X) / \psi$ is a splitting lattice. Hence if some prime quotient remained after collapsing quotients of (13), then this prime quotient would be separated by a bounded homomorphism onto a splitting lattice. Such lattices are known to be finite and semidistributive. Now it is easy to see that any finite, nontrivial semidistributive 


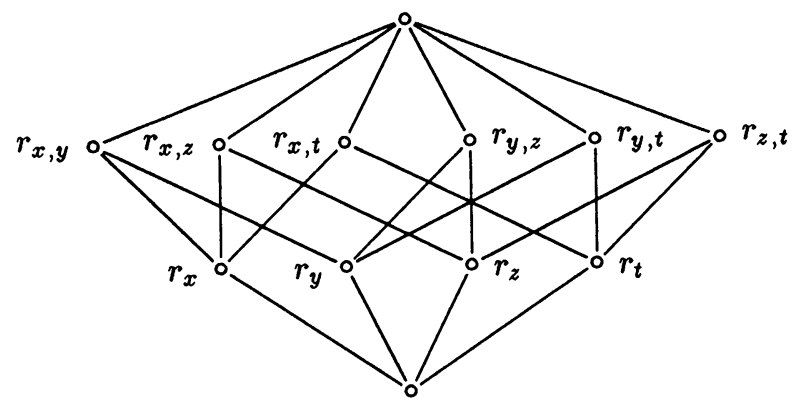

FIGURE 2

lattice has the two element lattice as a homomorphic image. Thus our finitely presented lattice would have the two element lattice as a homomorphic image. But the fact that all the quotients of (13) are collapsed, guarantees that it has no homomorphism onto the two element lattice.

However, this fact is not as strong as what we proved. Just because a finite presentation collapses all the existing covers does not guarentee that new covers will not arise. In fact this is exactly what happens when $|X|=3$. The lattice determined by collapsing all the prime quotients of $\mathbf{F L}(3)$ is $\mathbf{M}_{3}$, which does have covers.

This contrasts sharply with the situation in modular lattices. Every subdirectly irreducible, nondistributive modular lattice generated by a finite set $X$ satisfies the relations (10) (this follows from Wille's $D_{2}$-Lemma, see [14]). Many of these lattices are known to be bounded homomorphic images of $\operatorname{FM}(X)$, see [9], and hence the finitely presented modular lattice defined by the relations (10) has many covers.

\section{REFERENCES}

1. Peter Crawley and R. P. Dilworth, The algebraic theory of lattices, Prentice-Hall, Englewood Cliffs, N.J., 1973.

2. Alan Day, Splitting lattices generate all lattices, Algebraic Universalis 7 (1977), 163-169.

3. R. A. Dean, Free lattices generated by partially ordered sets and preserving bounds, Canad. J. Math. 16 (1964), 136-148.

4. Trevor Evans, The word problem for abstract algebras, J. London Math. Soc. 26 (1951), 654671.

5. Ralph Freese, Breadth two modular lattices, Proc. Univ. of Houston Latice Theory Conference, Univ. of Houston, 1973, pp. 409-451.

6. Ralph Freese and J. B. Nation, Finitely presented lattices, Proc. Amer. Math. Soc. 77 (1979), 174-178.

7. _ C Covers in free lattices, Trans. Amer. Math. Soc. 288 (1985), 1-42.

8. G. Grätzer, A. P. Huhn and H. Lakser, On the structure of finitely presented lattices, Canad. J. Math. 33 (1981), 404-411.

9. Ralph McKenzie, Equational bases and nonmodular lattice varieties, Trans. Amer. Math. Soc. 174 (1972), 1-43. 
10. Ralph McKenzie, George McNulty and Walter Taylor, Algebras, lattices, varieties, Vol. I, Wadsworth and Brooks Cole, Monterey, Calif., 1987.

11. J. C. C. McKinsey, The decision problem for some classes of sentences without quantifiers, J. Symbolic Logic 8 (1943), 61-76.

12. Ph. M. Whitman, Free lattices, Ann. of Math. (2) 42 (1941), 325-330.

13. _ Free lattices II, Ann. of Math. (2) 43 (1942), 104-115.

14. R. Wille, On free modular lattices generated by finite chains, Algebra Universalis 3 (1973), 131-138.

Department of Mathematics, University of Hawail, Honolulu, Hawail 96822 